\title{
Adopting Medical Computer Systems: The Perspectives of Physicians in a Poor Setting
}

\author{
Jerome Addah \\ Department of Community \\ Medicine, \\ School of Medicine and Health \\ Sciences \\ University for Development \\ Studies, \\ Tamale Ghana West Africa \\ Post Office Box TL 1883
}

\author{
Stephen Apanga \\ Department of Community \\ Medicine, \\ School of Medicine and Health \\ Sciences \\ University for Development \\ Studies, \\ Tamale Ghana West Africa \\ Post Office Box TL 1883
}

\author{
Mustapha Issahaku \\ Department of Community \\ Medicine \\ School of Medicine and Health \\ Sciences \\ University for Development \\ Studies, \\ Tamale Ghana West Africa \\ Post Office Box TL 1883
}

\begin{abstract}
The study sort to determine if physicians in poor settings were ready to use EMR systems: and also to find out which EMR system functional specifications and requirements would suit their practice. Statistical methods used in this study include univariate, bivariate and multivariate analytical techniques. From the univariate analysis we were able to unearth an existing culture of computer use among responding physicians, with the bivariate techniques we established that facilitate physician workflow and workload management were considered relevant by physicians. Results from the multivariate analysis indicated that systems with ubiquitous presence that were easily accessible, and those without any downtime had a predictive effect on a physician's decision of system capability relevance.
\end{abstract}

\section{Keywords}

Electronic medical records, medical compute system capabilities,

\section{INTRODUCTION}

Medical information systems that facilitate the collection , storage, and display of information on patients at health facilities are generally called Electronic Medical Record (EMR) systems; they provide a means to create legible and organized data repositories that facilitate access to individualized patient clinical information [1]. The intended purpose for them (EMRs) is to replace existing paper based medical record systems[1]. Paper based medical records have been in existence for generations, over this period of time they have taken up increasingly more facility space, while also causing delayed access to efficient medical care [2]. In contrast individualized patient clinical information are stored in repositories that are created electronically, thus enabling timely and ready access to this information by all providers in the health care chain, resulting in coherent and consistent patient care [3].Despite the high expectation and interest that has been generated in EMRs worldwide, widespread adoption rates are relatively low especially in poor settings; their adoption is generally problematic [4]For instance some physicians see their use as departing from their traditional working styles, others complain that EMR systems often require considerable skill in dealing with, while health facility managers protest the high initial cost of installation of EMR systems [5]. It has been suggested that the slow rate of adoption is an indication of strong resistance among physicians[6]. Such resistance among physicians is noteworthy because physicians constitute a major frontline user group, hence they are in a position to influence the use or otherwise of EMRs by other user groups within healthcare facilities[6]. As a result physicians have a great impact on the overall adoption levels of EMRs. It is therefore important to incorporate the opinions of physicians into any EMR adoption plans. It is however not clear what the opinions of physicians on EMR capabilities are in poor settings. In view of this, the objective of the current study is to find out from physicians which EMR capabilities they consider important to their practice and therefore would not patronize EMRs without those capabilities.

\section{METHODS AND MATERIALS}

This study is the result of a survey among practicing physician doctors at a Ghanaian Teaching Hospital affiliated to a Medical School using a questionnaire designed to investigate skills in ICT, and physician attitudes towards incorporating ICT into their practice and the medical school curriculum. Physicians who returned their completed questionnaires were regarded as having given their consent to participate in this study.

\subsection{Setting and Participants}

A cohort of 140 physicians of the Tamale Teaching Hospital was served questionnaires. The participating physicians were informed of the Purpose of the study, the requirement to complete a questionnaire, and the general content of the questionnaire. They were also told that their participation in the study was voluntary and that no personal identifiable information was going to be taken. The teaching hospital is located in Tamale the metropolitan capital of the northern region of Ghana. It is one of the third generations of teaching hospitals to be established in the country. The hospital is affiliated to the medical school of the University for Development Studies. At the moment, the hospital has no 
public access to computers for medical students, it however provides broadband internet access for staff and students, and computers are not available in the library for students to use, currently the hospital is the only clinical training site for medical education in northern Ghana.

\subsection{Survey Instrument}

The students were asked the following questions pertaining to: instructional methods, educational tools, ICT skill types: Basic (able to do basic word processing and use the internet), Intermediate (Have mastered the basics and have developed additional skills, including the use of different software programs), Advanced (Knowledgeable about hardware and software), ability to perform certain task with computers, frequency of computer use, they were also given a list of desired EMR system capabilities and then asked to judge if those capabilities were; Relevant (I would be much more likely to use a system with this capability, I would however not use a system that lack it.), Non- Relevant (my decision to use a system would be unaffected by the presence of this capability), I don't know ( the meaning or implication of this capability is not clear to me.). A drafted version of the questionnaire was administered to students $(n=100)$ in June, 2011. Internal reliability (Cronbach's alpha) obtained from combining items with ordinal responses was 0.82 (95\% CI)

for intra class correlation coefficient; 0.79 to 0.88 . The questionnaires were administered to the physicians who consented to participate in the study.

\subsection{Data Analysis}

Statistical analysis was performed using STATA (version 11.0, StataCorp. 2009). In order to identify responding physician's computer use habits, an analysis of frequencies of items derived from responses to questions related to frequent computer use was undertaken. Categorical variables relating to EMR system capabilities were analyzed using chi-square. Finally, a model to predict the occurrence of 'Relevant capability' was developed. To estimate the overall predicative effect of individual variables and desired capabilities, a categorical variable relevance with values (1'Relevant", 0 "Non Relevant") was selected as dependent variable), a binomial logit model was applied. The extent to which independent variables influenced the judgment of physicians in relation to the relevance or otherwise of a system capability was determined using the regression coefficients.

\section{RESULTS}

Overall 140 physicians received survey questionnaires, out of which 120 of them returned their questionnaires, resulting in a response rate of $85.7 \%$. The mean age for responding physicians was 29 years with a distribution of $29 \pm 1.4$ (mean \pm standard deviation). The physicians were mostly men, majority used a computer daily, while a small number said they don't use computers (Table 1).

Table1. Participant $(n=120)$ Characteristics and Demographics'

\begin{tabular}{|l|l|}
\hline & $\mathrm{n}(\%)$ \\
\hline Age & $29.0 \pm 1.4$ \\
\hline Gender & \\
\hline Female & $49(40.8 \%)$ \\
\hline Male & $61(50.8 \%)$ \\
\hline Frequency of computer use & \\
\hline Daily & $61(50.8 \%)$ \\
\hline Weekly & $20(16.7 \%)$ \\
\hline Rarely & $15(12.5 \%)$ \\
\hline Never & $14(11.7 \%)$ \\
\hline Training received in computers & \\
\hline medical school & $19(15.8 \%)$ \\
\hline residency or fellowship & $0(0.0 \%)$ \\
\hline Workshop or conference & $7(5.8 \%)$ \\
\hline Self-guided learning & $73(60.8 \%)$ \\
\hline None & $11(9.2 \%)$ \\
\hline
\end{tabular}

Majority of responding physicians acquired their proficiency in computer usage through self- learning $(60.0 \%)$, followed by training in computing while in medical school (15.8\%), a small minority $(9.2 \%)$ indicated they had not received any training in computing (Table1).

Table2- A contingency table describing physicians judgment on the relevance (Relevant and Non-Relevant) EMR system capabilities

\begin{tabular}{|c|c|c|c|}
\hline & $\begin{array}{l}\text { Relevant } \\
(\mathrm{n}=59)\end{array}$ & $\begin{array}{c}\text { Non-Relevant } \quad(\mathrm{n}=39 \\
)\end{array}$ & P- Value \\
\hline \multicolumn{4}{|l|}{ System Operational Capabilities } \\
\hline I can enter information in my own words and not need to know any special code & $49(83.1 \%)$ & $14(35.9 \%)$ & $<0.0001$ \\
\hline I can interact with the computer without using a keyboard & $42(71.2 \%)$ & $20(51.3 \%)$ & 0.0454 \\
\hline The system is always functioning. There is never any downtime & $44(74.6 \%)$ & $20(51.3 \%)$ & 0.0177 \\
\hline I can access the system at any place in the clinical setting & $51(86.4 \%)$ & $22(56.4 \%)$ & 0.0008 \\
\hline the system always responds to my queries in less than five seconds & $44(74.6 \%)$ & $23(59.0 \%)$ & 0.104 \\
\hline
\end{tabular}




\begin{tabular}{|c|c|c|c|}
\hline I can learn to use the system in less than two hours & $46(78.0 \%)$ & $18(46.2 \%)$ & 0.001 \\
\hline the system always displays Xrays and other images in less than 30 Seconds & $50(84.7 \%)$ & $24(61.5 \%)$ & 0.009 \\
\hline $\begin{array}{l}\text { the system can be implemented with no changes whatsoever to existing clinic } \\
\text { routines }\end{array}$ & $43(72.9 \%)$ & $22(56.4 \%)$ & 0.004 \\
\hline
\end{tabular}

In respect of which desired system operational capabilities (Table2) were considered by responding physicians as been relevant or not, $59.0 \%$ of responding physicians considered one or the other capability has been relevant (Table2).

Physicians who judged, a particular system capability to be relevant, mostly desired systems that were accessible anywhere within clinical settings $(86.4 \%)$, could display xrays and other images in less than 30 seconds $(84.7 \%)$, they could enter information in their own words without knowing any special codes $(83.1 \%)$, is easy to learn $(78.0 \%)$, systems without any downtimes (74.6\%), could be implemented without changes existing clinical routines $(72.9 \%)$, could be interacted with without using a keyboard (71.2\%) (Table2).

Table 3- EMR system capabilities with predictive effect on physician judgment on the relevance of system capabilities

\begin{tabular}{|l|l|l|l|}
\hline Variable & Estimated Coefficient & $\mathrm{P}>|\mathrm{z}|$ & {$[95 \%$ Conf. Interval] } \\
\hline $\begin{array}{l}\text { The system is always functioning. There is } \\
\text { never any downtime }\end{array}$ & -2.37 & 0.006 & $-4.068538 \quad-.6747923$ \\
\hline $\begin{array}{l}\text { I can access the system at any place in the } \\
\text { clinical setting }\end{array}$ & -2.05 & 0.003 & $-3.404659-.6904386$ \\
\hline
\end{tabular}

Two system operational capabilities were identified to be significantly associated with the relevance (Relevant or NonRelevant) of a system capability (Table3). We noticed a system that lacked the capability of been accessed anywhere within the clinical setting was significantly more likely not to

be patronized by physicians (Table3). Likewise, a system that did not have the capability of continuous functionality without

any downtimes also had a significant likelihood of not been used by physicians (Table3).

\section{DISCUSSION}

The results of this study suggest that a culture of computer exist among responding physicians this is indicated by the high proportion who said they use computers daily. This culture may have emerged from the teaching activities of responding physicians. Since the study site is a teaching facility, most of the study participants are involved in academic activities such as teaching, presentations at seminars workshops and conferences, most of them have had to use a computer to carry out this activities (such as preparing teaching slides, case studies, and assignments). The observed culture of computer use presents a suitable platform for the introduction of EMRs, since there is likely to be less resistance among physicians towards such a system because of their familiarity with computers. Studies have shown that EMR implementation success tends to be high among physicians in involved in teaching and those in large hospitals [7],

It was also revealed through this study that most responding physicians acquired their training in computer usage through self-tuition, this suggest a high level of enthusiasm among them to develop their competence in using computers. A caveat in this finding however is that because most responding physicians acquired their knowledge and skill in computer usage through self-tuition, their level of competence may not be as high as desired. It has been shown that one of the major reasons for physician resistance to EMRs is insufficient knowledge and computer skills [8-11]. The observed low level of knowledge and skill may be due to inadequate integration of information communication technology into the curricula of most medical programs [12]. EMR providers often gloss over this factual reality, and fail to incorporate it in their programming, resulting in the development of systems that require physicians to have advanced computer skills such as good typing skills for instance, to be able to enter patient medical information, notes, and prescriptions, the lack of this skills have led to the emergence of a new type of medical error called typos in facilities where EMRs are been implemented [13]. The lack of computer skills is not peculiar to physicians alone, but also prevalent among the other staff categories in health facilities [3]. Within our context, this general lack of skills may be mitigated by harnessing the observed enthusiasm for acquiring computer skills among physicians, to provide them with skills development opportunities.

Providing physicians with opportunities to develop and improve their knowledge and skills in the use of computers, may not be sufficient to encourage patronage of EMR systems among them, in addition to such efforts EMR systems need to be designed to meet the functional specifications and requirements of physicians. Results from this study suggest that such functional requirements may include a system that can be easily accessed anywhere within the clinical setting, and systems with continuous functionality without any downtimes. The observed desire for systems with ubiquitous presence in clinical settings that enable easy access may be 
motivated by the need for mobile patient care (the ability to respond to patient needs on the go), and the ability to respond to emergencies timely. The expressed need for systems that are always functioning without any downtimes may be due to on one hand the need to protect patient life's and apprehensions influenced by reports of lack of follow-up vendor support upon implementation and usability problems that usually cause frequent downtimes.

Many physicians bemoan the absence of after sale vendor support services such as poor follow - ups with technical issues, and a general lack of training and support for problems associated with EMRs [14]. The difficulties with vendor based training and support for technical issues is also echoed by Ludwick et al. [15]. Physicians perceive a need for proper technical training and support, and are therefore reluctant to use EMRs without it [3]. Usability problems due to insufficient computer knowledge and skills by physicians and the inherent complexity of most EMR systems result in frequent system downtimes, Miller and Sim observe that physicians consider EMRs difficult to use because of the multiplicity of screens, options, and navigation aids [15]. Such complexities create usability problems that result in frequent system downtimes, to avert such undesirable ends physicians, may have to allocate time and effort to learn their EMR systems; in poor settings where physicians' time is such a scarce resources such demands may be elicit strong opposition from physicians.

Concerns among responding physicians that EMR systems would slow their workflow and increase their workloads was also evident in this study, it was noted that physicians consider systems with capabilities such as; been learnt in less than two hours, and been able to display x-ray and other images in less than 30 seconds to be relevant. The suggested complexity of EMR and the lack of adequate computer skills among physicians give prominence to this findings, notwithstanding that it has been argued that the observed complexity of EMR systems by physicians may actually be due to their lacking, the requisite computer skills, consequently learning difficulties may actually be due to deficiencies in computer skills and not that EMR systems are inherently complex [3].

Most researches erroneously conclude that data entry is a problem for EMR users [15-18]. Such conclusions may be influenced by sentiments often made by physicians that data entry in EMR systems is time consuming and cumbersome [18]. Similar sentiments were expressed by responding physicians in the current study. We are inclined to believe to believe that such sentiments may actually be due to physicians possessing insufficient computer skills [3].

\section{CONCLUSION}

This study has unearthed favourable factors foster the introduction of EMR systems into medical teaching facilities in poor settings, among such factors is the existence of a culture of computer use among physicians. The results of this study may also help EMR system developers understand the system capabilities that are considered relevant by physicians in poor settings, so that they incorporate them into their systems programming.

\section{ACKNOWLEDGMENTS}

The students who willing participated in this study are greatly indebted,

\section{REFERENCES}

[1] McLane S. Designing an EMR Planning Process Based on Staff Attitudes Toward and Opinions About Computers in Healthcare. Computers Informatics Nursing 1 2005;.23: 85-92.

[2] Da've D. Benefits and Barriers to EMR Implementation. Caring. 12004;23: :50-51.

[3] Boonstra A, Broekhui, M. RESEARCH ARTICLE Open Access Barriers to the acceptance of electronic medical records by physicians from systematic review to taxonomy and interventions. BMC Health Services Research 12010: 10 - 23.

[4] Davidson E, Heslinga, D. .. Bridging the IT Adoption Gap for Small Physician Practices: An Action Research Study on Electronic Health Records. Information Systems Management 12007;; 24: 15-28.

[5] DesRoches C, Campbell EG, Rao SR, Donelan K, Ferris TG, Jha A, Kaushal R, Levy DE, Rosenbaum S, Shield AE, Blumenthal D. Electronic Health Records in Ambulatory Care - A National Survey of Physicians. New England Journal of Medicine. 12008;;359(1):: 50 60.

[6] Meinert D. Resistance to Electronic Medical Records (EMRs): A Barrier to Improved Quality of Care. Issues in Informing Science \& Information Technology 1 2005;2:: 493-504.

[7] Fleurant M, Kell R, Jenter C, Volk LA, Zhang F, Bates DW, Simon SR. Factors associated with difficult electronic health record implementation in office practic. J Am Med Inform Assoc. 2012 Jul-Aug; 12012;19: 5414.

[8] Vishwanath A, Scamurra SD. 1. Health Informatics Journal. ;. Barriers to the Adoption of Electronic Health Records: Using Concept Mapping to Develop a Comprehensive Empirical Mode. 12007;13: :119-134.

[9] Terry A, Thorpe CF, Giles GG, Brown JB, Harris SB, Reid GJ, Thind A, Stewart M. Canadian Family Physician. Implementing Electronic Health Records. 1 2008;;54:: 730-736.

[10] Simon, SR, Kalshal R, Cleary PD, Jenter CA, Volk LA, Oray EJ, Burdick E, Poon EG, Bates DW. . ;. Physicians and Electronic Health Records: A Statewide Survey. Archives of Internal Medicine. 12007;167: 507-512.

[11] Jha A, Bates DW, Jenter C, Orav EJ, Zheng J, Cleary P, Simon R. . . Electronic Health Records: Use, Barriers and Satisfaction Among Physicians Who Care For Black and Hispanic Patients. Journal of Evaluation in Clinical Practice. 12009;;15: 158-163.

[12] Meade B, Buckley D, Boland M. What Factors Affect the Use of Electronic Patient Records by Irish GPs? International Journal of Medical Informatics 1 . 2009;.78: :551-558.

[13] Pizziferri L KA, Volk LA, Honour MM, Gupta S, Wang S, Wang T, Lippincott M, Li Q, Bates DW. .;. . Primary Care Physician Time Utilization Before and After Implementation of an Electronic Health Record: A timemotion Study. Journal of Biomedical Informatics 1 2005;38: 176-188. 
[14] Randeree E. Exploring Physician Adoption of EMRs: A Multi-Case Analysis. Journal of Medical System. 12007;31: 489-496.

[15] Ludwick DA DJtp. Primary Care Physicians' Experience with Electronic Medical Records: Barriers to Implementation in a Fee-for-Service Environmen. International Journal of Telemedicine and Applications 1 2009.: 853-524.

[16] Valdes I, Kibbe DC, Tolleson G, Kunik ME, Petersen LA. . ; Barriers to Proliferation of Electronic Medical Records. Informatics in Primary Care 12004;12: 3-9.
[17] Menachemi N, Langley A, Brooks RG. . . The Use of Information Technologies Among Rural and Urban Physicians in Florida. Journal of Medical Systems 12007; 31: 483-488.

[18] Loomis G, Ries S, Saywell RM, Thakker NR. ;. If Electronic Medical Records Are So Great, Why Aren't Family Physicians Using them? Journal of Family Practice. 12002;51: 